\title{
Retorno Deportivo Luego de Reconstrucción Primaria de Ligamento Cruzado Anterior con Injerto Hueso-Tendón Patelar-Hueso Autólogo: Análisis de Factores Relacionados
}

\section{Return to Play after Anterior Cruciate Ligament Reconstruction with Bone-Patellar Tendon-Bone Autograft: Analysis of Related Factors}

\author{
A. Pakuts ${ }^{1,2}$ L. Martin ${ }^{3(0)}$ J. Contador ${ }^{30}$ \\ ${ }^{1}$ Especialista en Traumatología y Ortopedia, Facultad de Medicina, \\ Pontificia Universidad Católica de Chile, Chile \\ ${ }^{2}$ Centro Médico Ossis, La Serena, Chile \\ ${ }^{3}$ Interno, Facultad de Medicina, Pontificia Universidad Católica de \\ Chile, Chile \\ Rev Chil Ortop Traumatol 2020;61:2-10.
}

\begin{abstract}
Keywords

- anterior cruciate ligament

- reconstruction

- return to sports

- kinesiophobia

- bone patellar tendon bone

Objective Analyze return to sports and related factors after primary anterior cruciate ligament reconstruction.

Methods Observational descriptive study. 173 patients operated by the same surgeon and standardized technique (patellar autograft) who answered a questionnaire at least 12 months post procedure were included. Questionnaire included Subjective IKDC, Tegner activity level (Pre and Postoperative) and questions elaborated by the group.

Results Mean age was 30.8 years, $85 \%$ were men, $73 \%$ practiced soccer and median postoperative IKDC was 71 . Follow up until questionnaire response was 28 months. Males had a better return to sports than females ( $70 \%$ vs $48 \%, p=0.037$ ). Tegner preinjury level was 5 vs 4.3 postoperative, $(p<0.001)$. Return to sports was $67 \%$ according to Tegner scale and $66 \%$ by self-assessment. Return to similar previous activity level was $57 \%$ by Tegner scale but $24 \%$ by direct questions. Of those patients, $51 \%$ have fear of reinjury and $26 \%$ by reasons other than knee or surgery. We didn't find association between meniscal injuries and return to sports rate. Patients with chondral injuries had lower rates in return to sports ( $35 \%$ vs $60 \%, p=0.002$ ). Subjects that returned to sports had higher IKDC scores (73.5 vs $64.3, p<0.001)$.

Conclusions We found $67 \%$ return to sports and $57 \%$ to the preinjury level. Positive return to sports factors were male sex, absence of chondral injury and better functional outcome. Psychological factors such as fear of injury is frequent in patients who don't achieve previous levels of activity.
\end{abstract}

Address for correspondence Andrés Pakuts, La Serena, Chile (e-mail: andrespakuts@gmail.com). received

August 7, 2019

accepted

March 10, 2020
DOI https://doi.org/

$10.1055 / \mathrm{s}-0040-1709721$. ISSN $0716-4548$.
Copyright $\odot 2020$ by Thieme Revinter

Publicações Ltda, Rio de Janeiro, Brazil
License terms

c) $(\oplus) \$$ 


\section{Resumen}

\section{Palabras Clave}

- ligamento cruzado anterior

- reconstrucción

- retorno deportivo

- temor a nueva lesión

- hueso tendón hueso patelar
Objetivos Analizar el retorno deportivo y factores asociados tras la reconstrucción primaria de ligamento cruzado anterior (LCA).

Métodos Estudio observacional descriptivo. Se incluyeron 173 operados entre 2014 y 2017 por el mismo cirujano, los cuales contestaron un cuestionario al menos 12 meses después de la cirugía. El cuestionario incluye IKDC subjetivo, Tegner activity level (pre y post operatorio) y preguntas de elaboración propia.

Resultados La edad promedio es 30,8 años, el $85 \%$ son hombres, el $73 \%$ practicaba fútbol y la mediana del IKDC fue 71. La media de meses hasta responder el cuestionario fue de 28 meses. Tegner pre-lesión promedio fue de 5 vs 4,3 postoperatorio, $p<0,001$. Según la escala Tegner el 57\% retorna al mismo nivel previo, sin embargo, de acuerdo con el cuestionario propio solo el $24 \%$ lo haría. De ese subgrupo, el $51 \%$ tiene temor a lesionarse de nuevo y el $26 \%$ reporta razones no relacionadas a la rodilla. No encontramos asociación entre lesiones meniscales y la tasa de retorno. Aquellos que retornan tienen menor prevalencia de lesiones condrales ( $35 \%$ vs $60 \%, p=0,002$ ). Los pacientes que retornaron tuvieron un IKDC superior (73,5 vs $64,3, p<0,001)$. El sexo masculino tiene una tasa de retorno de $70 \%$ vs $48 \%$ de su contraparte femenina $(p=0,037)$.

Conclusiones El $67 \%$ retorna al deporte y el 57\% lo hace al mismo nivel. Factores positivos relacionados al retorno fueron sexo masculino, ausencia de lesión condral y mejor resultado funcional. Factores psicológicos con el miedo a lesionarse de nuevo son frecuentes en pacientes que no recuperan el nivel previo.

\section{Introducción}

Los pacientes que presentan rotura de ligamento cruzado anterior (LCA) frecuentemente son sometidos a una reconstrucción de LCA con el objetivo de recuperar la función y estabilidad de la rodilla. El retorno a la participación en actividad deportiva es un resultado importante cuando evaluamos el resultado de una cirugía de reconstrucción de LCA. $^{1}$

Estudios recientes señalan que la relación entre el resultado funcional alcanzado no necesariamente tiene una relación estrecha con la capacidad de volver a la actividad deportiva y menos aún en recuperar el mismo nivel previo a la lesión. ${ }^{2-4}$

Si bien comúnmente se consideraba que luego de una reconstrucción de LCA el retorno al deporte era un hecho común, estudios específicamente dirigidos a ese punto, indican que el retorno al mismo nivel de deporte no sería tan frecuente como antiguamente se afirmaba, variando entre 44 y $80 \% .^{5-7}$ Una revisión sistemática internacional reporta cifras de retorno al nivel deportivo previo cercanas al $63 \%^{8}$ sin embargo, estudios que evalúen particularmente ese punto en la población de nuestro país son escasos.

Los factores que determinan la tasa de retorno a la actividad son variados $y$ no han sido ampliamente estudiados. En la literatura se describen una serie de ellos, incluyendo múltiples factores tanto de la rodilla, estabilidad, función del cuádriceps, dolor y lesiones asociadas, como de factores propios del paciente tales como cambios en estilo de vida, kinesofobia y otros factores psicológicos. ${ }^{1,9-11}$
Este trabajo realizado en pacientes sometidos a la reconstrucción de LCA, tiene como objetivo determinar el retorno deportivo en la población estudiada, su relación con factores propios del paciente y las lesiones asociadas a la rotura del LCA.

\section{Material y Métodos}

Estudio observacional descriptivo realizado en centro único. Se incluyen pacientes operados de reconstrucción de ligamento cruzado anterior entre enero de 2014 y diciembre de 2017, que cumplen con los siguientes criterios: cirugía primaria de rotura aislada del LCA en los que se utilizó la técnica de injerto con hueso-tendón-hueso (HTH) autólogo, que tengan una evolución mínima de 12 meses desde su cirugía, y que fueron operados por el mismo primer cirujano. Se excluyen pacientes operados con otros tipos de injerto (isquiotibiales, tendón cuadricipital o aloinjerto), inestabilidades multiligamentarias, pacientes con fisis abierta y cirugías de revisión realizada o pendiente. La muestra inicial consistió en 251 pacientes que cumplieron esos criterios.

Se aplica un cuestionario al término del seguimiento que incluye "IKDC subjective knee form" y el score "Tegner Activity Level", validados internacionalmente ${ }^{12-14} ; \mathrm{y}$ en forma adicional se agrega un cuestionario con preguntas específicas, algunas dirigidas y otras abiertas con la finalidad de establecer apreciación personal respecto al retorno al deporte (-Anexo 1). Del total, 176 casos contestan el cuestionario, de los cuales 3 pacientes presentan rotura del injerto durante el seguimiento, por lo que son excluidos del 
estudio en base a nuestros criterios establecidos (tasa de rerotura $1,7 \%$ ). Se incluyen finalmente 173 pacientes que contestaron el cuestionario (69\% de muestra inicial), el cual fue aplicado con un seguimiento promedio de 28 meses postoperatorio (rango 12 a 58 meses).

La reconstrucción fue realizada con técnica estandarizada utilizando injerto autólogo del tercio central del tendón patelar de $10 \mathrm{~mm}$ de ancho, con posicionamiento de túneles óseos y apoyo de radiografía intraoperatoria. La fijación femoral y la tibial fue realizada con tornillos interferenciales de titanio, el tensionamiento manual del injerto a $20^{\circ}$ de flexión y en todos los pacientes, relleno de injerto óseo esponjoso en zona dadora de rótula obtenido del brocado del túnel femoral y del calibrado de los bloques óseos del injerto.

Se analizaron las siguientes variables: edad, sexo, mecanismo de lesión, deporte realizado, tiempo transcurrido entre la lesión y la cirugía, lesiones condrales y meniscales asociadas, IKDC subjetivo, Tegner Activity Level (pre y post operatorio). Se estudia la relación de esas variables con la capacidad de volver a la actividad física y el nivel de retorno deportivo alcanzado.

Se definió como retorno a deporte o retorno a la actividad física si logra un nivel de actividad mayor a 4 en la escala de Tegner y como retorno al mismo nivel deportivo si recuperaban el mismo o mayor nivel. En forma adicional, se aplicó una pregunta especialmente dirigida a la autopercepción del paciente sobre su nivel de retorno.

En el análisis estadístico se utilizó test Chi-cuadrado y exacto de Fisher para variables paramétricas y test de medianas de mood o mann-whitney para variables no paramétricas con significancia menor al 5\%. Se probó la normalidad con el test Anderson-Darling y KolmogorovSmirnov. En caso de no existir normalidad, se utilizó la mediana para determinar diferencias. El intervalo de confianza (IC) de las proporciones fue calculado con el método exacto para proporciones.

\section{Resultados}

Del total de 173 pacientes, 148 son del sexo masculino (86\%) con edad promedio de 30,8 años (DS 10,3, rango 14-60). Se encontraron diferencias al comparar el retorno al deporte entre mayores y menores de 30 años (74,4\% (IC 63,8-83,2\%) vs $59,7 \%$ (IC $48,7-70,1 \%$ ), respectivamente, $p=0,039$ ). En relación al sexo, la tasa de retorno al deporte es mayor en el sexo masculino (70,2\% (IC $62,2-77,5 \%$ ) vs $48 \%$ (IC 27,7-68,7), valor $p=0,037$ ), sin embargo, no encontramos tal diferencia si analizamos los que retornaron al mismo nivel deportivo (56\% (IC $47,7-7-2 \%$ ) vs $60 \%$ (IC $38,6-78,9 \%$, respectivamente con valor $p=0,712$ ).

El mecanismo de la lesión (disponible en 126 pacientes del total) fue torsional indirecto, en $91 \%$ de los casos, seguido por lesiones por contacto directo (6\%), y mecanismo mixto (3\%).

Respecto a los deportes realizados, el $73 \%$ practica fútbol y un $45 \%$ realiza más de un deporte ( - Tabla 1 ). En relación a la frecuencia de la actividad física previo a la lesión, realizan actividad deportiva 3,4 veces a la semana en promedio. Al final del seguimiento, se aprecia una disminución de la
Tabla 1 Descripción de la serie de 173 pacientes

\begin{tabular}{|l|l|l|}
\hline Categoría & Variable & Resultados \\
\hline $\begin{array}{l}\text { Tipo de deporte realizado } \\
\text { antes de la lesión. }\end{array}$ & Fútbol & $127(73 \%)$ \\
\hline & Ciclismo & $28(16 \%)$ \\
\hline & Running & $26(15 \%)$ \\
\hline & Baloncesto & $18(10 \%)$ \\
\hline & Gimnasio & $14(8 \%)$ \\
\hline & Motocross & $10(6 \%)$ \\
\hline & Voleibol & $8(5 \%)$ \\
\hline & Tenis & $8(5 \%)$ \\
\hline & Rugby & $8(5 \%)$ \\
\hline & $>1$ deporte & $81(47 \%)$ \\
\hline & Otros & $39(22 \%)$ \\
\hline
\end{tabular}

*Incluye deportes con número de pacientes menor a 8 en frecuencia: Acondicionamiento físico, Atletismo, Baile, BMX, Bodyboard, Boxeo, Break Dance, Caminata, Cheerleader, Dirt Jump, Entrenamiento Funcional, Escalada, Esqui, Handball, HIIT, Karate, Mountain Bike, Natación, Padel, Patín, Pesas, Sandboard, Skate, Spinning, Surf, Taekwondo, Trekking y TRX.

frecuencia de actividad deportiva, bajando a una media de 2,4 veces por semana.

Según la escala de Tegner, el nivel preoperatorio promedio es 5 . Un $43 \%$ tenía una actividad física que puede considerarse de mayor demanda (Tegner 6 o mayor) y un $9 \%$ presentan un nivel competitivo, de los cuales un $2 \%$ corresponden a nivel profesional. Después de la cirugía, el nivel presentó un promedio de 4,3. (-Tabla 2). Del total de 173 pacientes, un 67\% (116 pacientes), volvió a realizar actividad deportiva con Tegner mayor o igual a 4 y un $57 \%$ (98 pacientes), mantiene un nivel de actividad similar o mayor a la pre-lesión.

Al comparar esos resultados según Tegner, con las preguntas agregadas de autopercepción específicamente dirigidas a ese punto, el resultado fue que un 66\% (114 pacientes) retornan a la actividad deportiva, pero un $24 \%$ (59 pacientes) consideran que lo hacen al mismo o mayor

Tabla 2 Tegner Activity Level Pre vs Postoperatorio $(n=173)$

\begin{tabular}{|l|l|l|}
\hline Tegner Level & Preoperatorio & Postoperatorio \\
\hline 1 & $5(3 \%)$ & $5(3 \%)$ \\
\hline 2 & $10(6 \%)$ & $21(12 \%)$ \\
\hline 3 & $15(9 \%)$ & $31(18 \%)$ \\
\hline 4 & $48(28 \%)$ & $51(29 \%)$ \\
\hline 5 & $20(11 \%)$ & $21(12 \%)$ \\
\hline 6 & $39(22 \%)$ & $24(14 \%)$ \\
\hline 7 & $21(12 \%)$ & $12(7 \%)$ \\
\hline 8 & $1(<1 \%)$ & $7(4 \%)$ \\
\hline 9 & $10(6 \%)$ & $1(<1 \%)$ \\
\hline 10 & $4(2 \%)$ & $1(<1 \%)$ \\
\hline
\end{tabular}


Tabla 3 Frecuencia según respuestas a la pregunta "Indique en la casilla qué deportes o actividad realiza DESPUÉS de la lesión”

\begin{tabular}{|l|l|}
\hline Descripción & Resultados \\
\hline Realizo el mismo deporte a mayor intensidad & $8(4,6 \%)$ \\
\hline Realizo el mismo deporte a igual intensidad & $34(19,6 \%)$ \\
\hline Realizo el mismo deporte a menor intensidad & $72(41,6 \%)$ \\
\hline No he vuelto a realizar el mismo deporte & $59(34,2 \%)$ \\
\hline TOTAL: & $173(100 \%)$ \\
\hline
\end{tabular}

Tabla 4 Subgrupo de pacientes que indica realizar el mismo deporte a una menor intensidad o no volver a realizar el mismo deporte, n. $131^{*}$

\begin{tabular}{|l|l|}
\hline Descripción & Resultados \\
\hline Temor a lesionarse de nuevo & 67 \\
\hline Dolor durante el deporte & 23 \\
\hline $\begin{array}{l}\text { Sensación de inestabilidad } \\
\text { durante el deporte }\end{array}$ & 31 \\
\hline $\begin{array}{l}\text { Otro tema no relacionado } \\
\text { a la rodilla: carga laboral, } \\
\text { académica, otros proyectos, etc. }\end{array}$ & 34 \\
\hline
\end{tabular}

*Se aceptaron respuestas múltiples.

Tabla 5 Prevalencia de Lesiones Asociadas, $\mathrm{n}$ total $=173$

\begin{tabular}{|l|l|l|}
\hline Categoría & Descripción & Resultados \\
\hline Meniscal & Total: & $146(84,5 \%)$ \\
\hline & Externo & $112(65 \%)$ \\
\hline & Interno & $95(55 \%)$ \\
\hline & Bilateral & $61(35,3 \%)$ \\
\hline & Total: & $75(43 \%)$ \\
\hline & Externo & $25(14,5 \%)$ \\
\hline & Interno & $56(32,4 \%)$ \\
\hline & Bilateral & $10(5,8 \%)$ \\
\hline & Patelofemoral & $20(11,5 \%)$ \\
\hline
\end{tabular}

Tabla 6 Análisis por Variable según retorno al deporte y al mismo nivel, n=173

\begin{tabular}{|l|l|l|l|}
\hline & Retorno Deportivo & Valor-p \\
\hline & Si & No & \\
\hline Retorno al deporte (Tegner $>=4):$ & $116(67 \%)$ & $57(33 \%)$ & N/A \\
\hline -Lesión Condral (+) & $35 \%(I C 26,6-44,7 \%)$ & $60 \%(I C ~ 45,8-72,4 \%)$ & $p=0,002^{*}$ \\
\hline -Lesión Meniscal (+) & $83 \%(I C 74,6-89,1 \%)$ & $88 \%(76,3-95 \%)$ & $p=0,398^{*}$ \\
\hline Retorno a nivel (Tegner > Previo): & $98(57 \%)$ & $75(43 \%)$ & N/A \\
\hline -Lesión Condral (+) & $34 \%(I C 24,9-44,7 \%)$ & $56 \%(I C ~ 44-67,4 \%)$ & $p=0,003^{*}$ \\
\hline -Lesión Meniscal $(+)$ & $84 \%(74,8-90 \%)$ & $85 \%(I C 75,2-92,4 \%)$ & $p=0,766^{*}$ \\
\hline
\end{tabular}

Abreviatura: N/A, No Aplica.

*Prueba Chi-cuadrado.
Tabla 7 Mediana de latencia quirúrgica en meses según tipo de lesión

\begin{tabular}{|l|l|l|l|}
\hline $\begin{array}{l}\text { Tipo de } \\
\text { lesión }\end{array}$ & Presente & Ausente & Valor-P \\
\hline Meniscal & $2,4($ IC 2,1-3,3) & $4($ IC 2,1-9,6) & $P=0,174$ \\
\hline Condral & 4,4 (IC 2,2-11) & $2,3($ IC $1,8-2,96)$ & $P=0,071$ \\
\hline
\end{tabular}

nivel (-Tabla 3). En el 76\% restante (131 pacientes) que considera bajar la intensidad o cambiar de deporte, aparece el temor a una nueva lesión como el factor más común, presente en el $51 \%$ de los pacientes de ese subgrupo. Por otro lado, 34 pacientes señalaron que el motivo del no retorno fueron temas no relacionados a su rodilla o su cirugía, sino más bien factores familiares, laborales y otros (-Tabla 4).

Nuestra serie de 173 pacientes presentó una mediana de IKDC de 71,2 (IC 68,9-72,4) con un rango de 34 a 83 puntos. Se encontró una diferencia significativa tanto para pacientes con retorno (73,5 (IC 72,3-74,7) vs 64,3 (IC 60,5-67,8), respectivamente con $p<0,001$ ) como los que lo hicieron al mismo nivel (73,5 (IC 71,2-74,7) vs 66,6 (IC 63,8-69,4), respectivamente con $p<0,001$ ).

El tiempo transcurrido entre la lesión y la cirugía presentó un amplio rango desde 10 días hasta un caso que tenía 26 años de antigüedad de la lesión, con una mediana de 2,6 meses. Al evaluar la relación entre el retorno deportivo y el tiempo de latencia a la cirugía, no encontramos diferencias (2,6 (IC 2,2-3,7) vs 2,3 meses (IC $1,9-7,6)$, respectivamente con $p=0,780$ ).

En relación a las lesiones asociadas, el 84,5\% (146 casos) presentó alguna lesión meniscal. Se pesquisaron lesiones condrales en 75 pacientes (43\%), su distribución se detalla en la - Tabla 5. Los pacientes con lesiones condrales presentaron diferencias significativas de retorno deportivo. No se encontraron diferencias al comparar el retorno deportivo con presencia de lesión meniscal (-Tabla 6).

No hubo diferencias en la frecuencia de lesiones meniscales o condrales al comparar con la latencia de la cirugía ( - Tabla 7).

\section{Discusión}

Una de las preguntas más comunes de un individuo que sufre una rotura de LCA, es si podrá volver a realizar su deporte y si 
lo hará al mismo nivel de desempeño. Al evaluar los resultados de participación deportiva, en primer lugar, es importante especificar el nivel de la actividad deportiva. Pueden clasificarse en la capacidad de volver a cualquier deporte, al mismo nivel deportivo pre-lesión y a nivel competitivo. En forma general, los estudios publicados en grupos de pacientes generales muestran un retorno deportivo entre un $44 \%$ y un $92 \% .^{5-7,15-21}$

Una revisión sistemática y un metaanálisis publicados en 2014 mostró que un $81 \%$ de 4837 individuos (en 57 trabajos) volvía a realizar algún deporte, mientras que un 65\% de 2663 atletas (en 50 estudios) retornaba al mismo nivel deportivo previo a la lesión. Al analizar deportistas de élite, un 55\% retornó a un nivel competitivo (1338 pacientes en 10 estudios $)^{7}$ Sin embargo, sólo un $38 \%$ se mantuvo al mismo nivel a los 2 años de su cirugía. Nuestra serie, formada en gran parte por atletas recreacionales, mostró un $67 \%$ de retorno a alguna actividad deportiva y un $57 \%$ al mismo nivel de acuerdo con la escala de Tegner. Si la definición de retorno al deporte se determinara como realizar "cualquier deporte," se incluirían pacientes en nivel 3 de Tegner lo cual elevaría las tasas de retorno deportivo de nuestro grupo a un $85 \%$. Ese factor es fundamental al comparar los distintos trabajos de retorno deportivo. En una revisión sistemática, Anderson concluye la falta de uniformidad en el criterio de retorno al deporte y que un $40 \%$ a un $65 \%$ de los estudios, simplemente no reportan cuáles fueron los criterios utilizados. ${ }^{22}$

En nuestro trabajo, si comparamos las tasas de retorno al deporte, vemos que el retorno deportivo según Tegner y según las preguntas de autopercepción son similares (67\% y $66 \%$, respectivamente), pero al evaluar los que logran un nivel deportivo similar al prelesional, se obtiene un $57 \%$ según la escala de Tegner, pero sólo un 24\% tiene la percepción personal de realizar deportes al mismo nivel. Esa diferencia importante no ha sido adecuadamente abordada en la literatura, sin encontrar reportes de ese aspecto y que son fundamentales al momento de determinar los resultados de la cirugía.

El retorno está determinado por múltiples variables tanto intrínsecas como extrínsecas del paciente. Está descrito que atletas jóvenes, sexo masculino, deportistas de élite y una respuesta psicológica favorable, tienen mayores niveles de retorno al nivel pre lesional. ${ }^{7}$ Sandon nos presenta un estudio en el que sólo el 54\% de 205 pacientes tuvieron retorno deportivo. ${ }^{20}$ Sus resultados demuestran que el sexo femenino, daño condral y el dolor durante la actividad deportiva, son factores negativos independientes en el retorno después de una reconstrucción de LCA y estando los 3 factores presentes sólo un $10 \%$ retornaron comparado con un $76 \%$ si ninguno de esos factores estaba presente.

En nuestro estudio, el sexo masculino y una edad menor a 30 años fueron factores positivos en cuanto al retorno a la actividad deportiva. Existen trabajos que concuerdan en que los pacientes de sexo masculino tienen mayores posibilidades de retorno al mismo nivel deportivo recreacional (OR 1,4) y competitivo (OR 1,7). ${ }^{23,24}$ Nuestro estudio concuerda en que el sexo masculino tiene mayor frecuencia de retorno al deporte, sin embargo, la asociación encontrada es relativa a qué definición de retorno deportivo se utiliza, al no encontrar diferencias en el grupo que retorna al mismo nivel deportivo. Por otro lado, existen estudios con resultados contradictorios respecto a la diferencia entre géneros, algunos afirman que la diferencia en la tasa de retorno podría deberse a que las mujeres requieren más tiempo para alcanzar el nivel deportivo pre-lesión y que a largo plazo las diferencias no son significativas. ${ }^{1,25}$

Respecto a la edad y su influencia en la tasa de retorno, Beisher en su estudio de 270 casos, demuestra un $74 \%$ de retorno en adolescentes y un $63 \%$ en adultos. $^{5}$ Un metaanálisis con 1156 casos en niños y adolescentes concuerda con ese mayor retorno al deporte en ese grupo etario de $92 \%$ y de un $78 \%$ al mismo nivel. ${ }^{15}$ Esos hallazgos concuerdan con lo encontrado en nuestro estudio, pero sólo al definir como punto de corte los 30 años, lo que podría deberse a que ese grupo etario tiene más facilidades para perseverar en la actividad deportiva debido a menores compromisos en el ámbito familiar y laboral, sobre todo en deportistas recreacionales, como afirma Kay y col. ${ }^{15}$

Otro aspecto importante al evaluar el retorno es el tipo de deporte realizado. ${ }^{26}$ Nuestra serie en su mayoría está formada por deportistas aficionados que practican fútbol. En un estudio europeo prospectivo en futbolistas, Walden, muestra que un 86\% sigue jugando, pero sólo un 65\% al mismo nivel de desempeño a los 3 años de seguimiento. ${ }^{27}$ Shah y col., analiza la Liga Nacional de Futbol Americano (NFL) encontrando un 63\% de retorno deportivo. ${ }^{28}$ Plancher, en su serie de casos describe distintas tasas de retorno según el deporte realizado, llegando incluso hasta $100 \%$ en el caso de ciclistas y $80 \%$ para tenistas. ${ }^{29}$ En la misma línea, Warner reporta que es más probable el retorno al ciclismo y al trote, que a actividades con cambios bruscos de dirección y pivot, como lo es el fútbol. ${ }^{30}$ Lo anterior denota que el tipo de deporte realizado y las distintas exigencias tanto estáticas como dinámicas del deporte realizado, influyen en la tasa de retorno al deporte.

La relación entre el resultado funcional de los pacientes y el retorno deportivo es un hecho generalmente aceptado, pero últimamente ha sido cuestionada si existe una relación tan estrecha. Ross, no encuentra relación entre resultado funcional y retorno al deporte. ${ }^{31}$ Por otra parte, Ardern en su estudio, concluye que una rodilla con función normal tiene el doble de posibilidades de retorno a similar nivel deportivo. ${ }^{8}$ Similar hallazgo se demuestra en un estudio de Webster. ${ }^{4}$ Nuestro estudio, concuerda con esos estudios y la mayoría de las publicaciones, donde se demuestra una relación entre el resultado funcional evaluado a través del puntaje del IKDC, y la capacidad de retorno a cualquier nivel deportivo.

En nuestra serie, el daño condral se asocia a un menor retorno deportivo, lo cual concuerda con trabajos previos. ${ }^{20}$ Eso puede deberse a que el daño condral secundario produzca dolor al realizar actividad al igual que la articulación de un paciente que padece osteoartritis. El dolor y una rodilla sintomática está presente en más del $50 \%$ de los pacientes 
que no recuperan su nivel deportivo previo y se asocia a peores resultados en las escalas que miden el estado funcional. ${ }^{1}$

Respecto a la relación entre las lesiones meniscales asociadas y el retorno deportivo, no encontramos una relación directa con la presencia de lesión. La alta prevalencia de ese tipo de lesiones, más del $80 \%$ en nuestro estudio, no pudimos explicarla al no encontrar diferencias respecto a la latencia quirúrgica y tal vez se relaciona a que se incluyen en nuestra estadística lesiones estables y parciales que no requirieron procedimientos quirúrgicos adicionales. Varios autores han descrito un riesgo aumentado de lesión meniscal con el retraso de la cirugía, ${ }^{16,32-36}$ llegando incluso a un $62 \%$ si la latencia quirúrgica supera los 5 meses, comparado con un $37 \%$ si la cirugía es precoz. ${ }^{32}$ En concordancia, otros autores han descrito que el retraso quirúrgico es un predictor importante tanto de lesiones meniscales como condrales. ${ }^{37,38}$ En cambio, Smith describe que no habría diferencias entre cirugía precoz y tardía para una serie de variables, incluyendo lesiones meniscales. ${ }^{39}$ En la misma línea, en un resumen de revisiones sistemáticas, Anderson reporta que existen resultados diversos, definiciones variadas de cirugía tardía o precoz en la literatura y se aconseja tener precaución con el retraso quirúrgico debido a que el impacto en el riesgo a largo plazo de lesiones meniscales y condrales no está bien determinado. 22,40

Hay un creciente interés y reportes de la importancia de los factores psicológicos en el retorno deportivo. Se ha identificado claramente, la influencia positiva de una respuesta psicológica adecuada en el retorno deportivo a

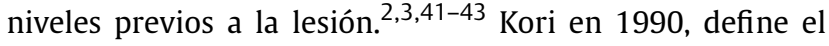
concepto de kinesiofobia como un miedo excesivo a la actividad física o el movimiento producto de un sentimiento de vulnerabilidad frente al dolor o lesionarse nuevamente. ${ }^{44} \mathrm{Hsu}$, publica una revisión dirigida al temor a una nueva lesión y concluye el impacto negativo tanto físico como psicológico en la función autoevaluada y en un menor retorno deportivo. ${ }^{42}$ En nuestra serie, el temor a una nueva lesión aparece como el factor más frecuente, presente en más de la mitad de los pacientes (51\%) que no retornan el nivel deportivo previo. En forma concordante, Flanigan en un estudio de 135 pacientes, encontró una tasa de temor o kinesiofobia del $52 \%{ }^{45}$ Medvecky describe que el aspecto psicológico y el temor podrían jugar un rol significativo a la hora de recuperar el nivel deportivo previo a la lesión sin tomar en consideración la estabilidad ni la fuerza en la articulación. ${ }^{46}$ Una revisión sistemática que incluyó 19 estudios y 2175 pacientes encontró que el 64,7\% refirió factores psicológicos como determinantes de un retorno inferior al previo a la lesión; dentro de esos pacientes el $76,7 \%$ reportó temor a una nueva lesión. ${ }^{47}$ Paterno, en un grupo de 40 pacientes encontró que los sujetos con temor significativo, evaluado a través de la "Tampa Scale of Kinesiophobia" tienen 13 veces más riesgo de volver a lesionarse dentro de 24 meses después de retornar al deporte. $^{3,48}$

Además del temor a una nueva lesión, distintos autores muestran factores como la falta de confianza, la inseguridad, la motivación y un cambio en las prioridades $y$ en las expectativas como aspectos primordiales que explican la conducta postoperatoria ante la actividad deportiva. ${ }^{3,49-51}$ Ese último punto, que vale la pena ser considerado antes de analizar fríamente las tasas de retorno deportivo, se refleja claramente en nuestro estudio en un significativo grupo de 34 pacientes (26\%), que simplemente no retornaron al deporte en el periodo estudiado por motivos no relacionados a su cirugía, ni a su rodilla.

Nuestro grupo de pacientes presentó una tasa de rerotura de $1,7 \%$ que corresponde a 3 pacientes. Una re-rotura de LCA después de una reconstrucción es un evento devastador para un deportista, afectando tanto física como psicológicamente. Hay múltiples factores que deben considerarse al analizar las causas de esa nueva lesión como errores técnicos en la cirugía, falla en la incorporación biológica del injerto, falla en la fijación, un nuevo traumatismo, o mecanismos mixtos. El porcentaje de rerotura o rotura contralateral se ha reportado entre $4 \%$ y $27 \%$ ocurriendo la mayor parte durante el primer año postoperatorio. ${ }^{52,53}$

Nuestro estudio presenta muchos hallazgos similares a los reportados por la literatura, reconociendo algunas limitaciones. Tuvimos una tasa de atrición de un $31 \%$, que son los pacientes que no contestaron el cuestionario y no pudieron ser incluidos. Ardern, presenta su estudio con una tasa de atrición del $41 \%{ }^{1}$ Un reducido grupo de pacientes del sexo femenino, que en nuestra serie es un $14 \%$ ( 25 pacientes), puede influir en un análisis estadístico adecuado. Otra limitación está determinada por la incorporación de algunas preguntas de elaboración propia no adecuadamente validadas lo cual es un hecho que se repite en diversos estudios. ${ }^{1,8,22}$ A la luz de los resultados obtenidos, en que destacamos la enorme importancia de los factores psicológicos, valdría la pena ahondar en ese aspecto en el futuro con escalas más adecuadas. $^{48}$

\section{Conclusiones}

El retorno al deporte es uno de los resultados más importantes luego de la cirugía de reconstrucción del LCA. Nuestro estudio presenta tasas de retorno a la actividad física de un $67 \%$ y a un nivel deportivo similar al prelesional de $57 \%$, comparables a los publicados en la literatura. Se determinaron como factores positivos para el retorno al deporte, el sexo masculino, la edad menor a 30 años, la ausencia de lesión condral y un mejor resultado funcional. Los factores psicológicos juegan un rol importante, destacando la frecuencia del temor a una nueva lesión tanto en la capacidad de retornar como en el nivel deportivo logrado.

Conflicto de Intereses

Los autores declaran no tener ningún conflicto de intereses. 


\section{Anexo 1 Preguntas adicionales}

1. En número de veces por semana ¿Cuántas veces a la semana realiza actividad física o deporte?

- Antes de la lesión: 01234567

- Después de la lesión: 01234567

2. Indique en la casilla qué deportes o actividad realizaba ANTES de la lesión:

- "Texto Libre"

3. Indique en la casilla qué deportes o actividad realiza DESPUÉS de la lesión: *

- En caso de seleccionar "No he vuelto a realizar el mismo deporte", indique en la casilla "otro" el deporte o actividad:

- Realizo el mismo deporte a mayor intensidad.

- Realizo el mismo deporte a igual intensidad.

- Realizo el mismo deporte a menor intensidad.

- No he vuelto a realizar el mismo deporte.

- Otro: "Texto Libre"

4. En caso de bajar el nivel de intensidad o cambiar de deporte ¿Porqué? Selecciona todos los que correspondan.

- No Aplica

- Temor a lesionarse de nuevo.

- Dolor al realizar ese deporte.

- Sensación de fallo o inestabilidad al realizar ese deporte.

- Por temas no relacionados a la rodilla (carga laboral, académica, otros proyectos, etc).

\section{Bibliografía}

1 Ardern CL, Taylor NF, Feller JA, Webster KE. Return-to-sport outcomes at 2 to 7 years after anterior cruciate ligament reconstruction surgery. Am J Sports Med 2012;40(01):41-48. Doi: $10.1177 / 0363546511422999$

2 Werner JL, Burland JP, Mattacola CG, Toonstra J, English RA, Howard JS. Decision to Return to Sport Participation After Anterior Cruciate Ligament Reconstruction, Part II: SelfReported and Functional Performance Outcomes. J Athl Train 2018;53(05):464-474. Doi: 10.4085/1062-6050-328-16

3 Paterno MV, Flynn K, Thomas S, Schmitt LC. Self-Reported Fear Predicts Functional Performance and Second ACL Injury After ACL Reconstruction and Return to Sport: A Pilot Study. Sports Health 2018;10(03):228-233. Doi: 10.1177/1941738117745806

4 Webster KE, Feller JA. Younger Patients and Men Achieve Higher Outcome Scores Than Older Patients and Women After Anterior Cruciate Ligament Reconstruction. Clin Orthop Relat Res 2017; 475(10):2472-2480. Doi: 10.1007/s11999-017-5418-2

5 Beischer S, Hamrin Senorski E, Thomeé C, Samuelsson K, Thomeé R. Young athletes return too early to knee-strenuous sport, without acceptable knee function after anterior cruciate ligament reconstruction. Knee Surg Sports Traumatol Arthrosc 2018;26(07):1966-1974. Doi: 10.1007/s00167-017-4747-8

6 Niederer D, Engeroff T, Wilke J, Vogt L, Banzer W. Return to play, performance, and career duration after anterior cruciate ligament rupture: A case-control study in the five biggest football nations in Europe. Scand J Med Sci Sports 2018;28(10):2226-2233. Doi: 10.1111/sms.13245

7 Ardern CL, Taylor NF, Feller JA, Webster KE. Fifty-five per cent return to competitive sport following anterior cruciate ligament reconstruction surgery: an updated systematic review and metaanalysis including aspects of physical functioning and contextual factors. Br J Sports Med 2014;48(21):1543-1552. Doi: 10.1136/ bjsports-2013-093398

8 Ardern CL, Webster KE, Taylor NF, Feller JA. Return to sport following anterior cruciate ligament reconstruction surgery: a systematic review and meta-analysis of the state of play. $\mathrm{Br} \mathrm{J}$
Sports Med 2011;45(07):596-606. Doi: 10.1136/ bjsm.2010.076364

9 Davies GJ, McCarty E, Provencher M, Manske RC. ACL Return to Sport Guidelines and Criteria. Curr Rev Musculoskelet Med 2017; 10(03):307-314. Doi: 10.1007/s12178-017-9420-9

10 Daruwalla JH, Greis PE, Hancock R, Xerogeanes JW; ASP Collaborative Group. Rates and Determinants of Return to Play After Anterior Cruciate Ligament Reconstruction in NCAA Division 1 College Football Athletes: A Study of the ACC, SEC, and PAC-12 Conferences. Orthop J Sports Med 2014;2(08): 2325967114543901. Doi: 10.1177/2325967114543901

11 Czuppon S, Racette BA, Klein SE, Harris-Hayes M. Variables associated with return to sport following anterior cruciate ligament reconstruction: a systematic review. Br J Sports Med 2014;48(05):356-364. Doi: 10.1136/bjsports-2012-091786

12 Tegner Y, Lysholm J. Rating systems in the evaluation of knee ligament injuries. Clin Orthop Relat Res 1985;(198):43-49. Doi: 10.1097/00003086-198509000-00007

13 Irrgang JJ, Anderson AF, Boland AL, et al. Development and validation of the international knee documentation committee subjective knee form. Am J Sports Med 2001;29(05):600-613. Doi: 10.1177/03635465010290051301

14 Briggs KK, Lysholm J, Tegner Y, Rodkey WG, Kocher MS, Steadman JR. The reliability, validity, and responsiveness of the Lysholm score and Tegner activity scale for anterior cruciate ligament injuries of the knee: 25 years later. Am J Sports Med 2009;37(05): 890-897. Doi: 10.1177/0363546508330143

15 Kay J, Memon M, Marx RG, Peterson D, Simunovic N, Ayeni OR. Over $90 \%$ of children and adolescents return to sport after anterior cruciate ligament reconstruction: a systematic review and meta-analysis. Knee Surg Sports Traumatol Arthrosc 2018;26(04):1019-1036. Doi: 10.1007/s00167-0184830-9

16 Lai CCH, Ardern CL, Feller JA, Webster KE. Eighty-three per cent of elite athletes return to preinjury sport after anterior cruciate ligament reconstruction: a systematic review with meta-analysis of return to sport rates, graft rupture rates and performance 
outcomes. Br J Sports Med 2018;52(02):128-138. Doi: 10.1136/ bjsports-2016-096836

17 Read CR, Aune KT, Cain EL Jr, Fleisig GS. Return to Play and Decreased Performance After Anterior Cruciate Ligament Reconstruction in National Football League Defensive Players. Am J Sports Med 2017;45(08):1815-1821. Doi: $10.1177 / 0363546517703361$

18 Dekker TJ, Godin JA, Dale KM, Garrett WE, Taylor DC, Riboh JC. Return to Sport After Pediatric Anterior Cruciate Ligament Reconstruction and Its Effect on Subsequent Anterior Cruciate Ligament Injury. J Bone Joint Surg Am 2017;99(11):897-904. Doi: $10.2106 /$ jbjs. 16.00758

19 Nwachukwu BU, Voleti PB, Berkanish P, et al. Return to Play and Patient Satisfaction After ACL Reconstruction: Study with Minimum 2-Year Follow-up. J Bone Joint Surg Am 2017;99(09): 720-725. Doi: 10.2106/jbjs.16.00958

20 Sandon A, Werner S, Forssblad M. Factors associated with returning to football after anterior cruciate ligament reconstruction. Knee Surg Sports Traumatol Arthrosc 2015;23 (09):2514-2521. Doi: 10.1007/s00167-014-3023-4

21 Rodríguez-Roiz JM, Caballero M, Ares O, Sastre S, Lozano L, Popescu D. Return to recreational sports activity after anterior cruciate ligament reconstruction: a one- to six-year follow-up study. Arch Orthop Trauma Surg 2015;135(08):1117-1122. Doi: 10.1007/s00402-015-2240-8

22 Anderson MJ, Browning WM III, Urband CE, Kluczynski MA, Bisson LJ. A Systematic Summary of Systematic Reviews on the Topic of the Anterior Cruciate Ligament. Orthop J Sports Med 2016;4(03): 2325967116634074. Doi: 10.1177/2325967116634074

23 Deaner RO, Geary DC, Puts DA, et al. A sex difference in the predisposition for physical competition: males play sports much more than females even in the contemporary U.S. PLoS One 2012; 7(11):e49168. Doi: 10.1371/journal.pone.0049168

24 Kondric M, Sindik J, Furjan-Mandic G, Schiefler B. Participation Motivation and Student's Physical Activity among Sport Students in Three Countries. J Sports Sci Med 2013;12(01):10-18

25 Thing LF. "Voices of the broken body." The resumption of nonprofessional female players' sports careers after anterior cruciate ligament injury. The female player's dilemma: is she willing to run the risk? Scand J Med Sci Sports 2006;16(05):364-375

26 Ellman MB, Sherman SL, Forsythe B, LaPrade RF, Cole BJ, Bach BR Jr. Return to play following anterior cruciate ligament reconstruction. J Am Acad Orthop Surg 2015;23(05):283-296. Doi: 10.5435/JAAOS-D-13-00183

27 Waldén M, Hägglund M, Magnusson H, Ekstrand J. ACL injuries in men's professional football: a 15-year prospective study on time trends and return-to-play rates reveals only $65 \%$ of players still play at the top level 3 years after ACL rupture. Br J Sports Med 2016;50(12):744-750. Doi: 10.1136/bjsports-2015-095952

28 Shah VM, Andrews JR, Fleisig GS, McMichael CS, Lemak LJ. Return to play after anterior cruciate ligament reconstruction in National Football League athletes. Am J Sports Med 2010;38(11): 2233-2239. Doi: $10.1177 / 0363546510372798$

29 Plancher KD, Steadman JR, Briggs KK, Hutton KS. Reconstruction of the anterior cruciate ligament in patients who are at least forty years old. A long-term follow-up and outcome study. J Bone Joint Surg Am 1998;80(02):184-197

30 Warner SJ, Smith MV, Wright RW, Matava MJ, Brophy RH. Sportspecific outcomes after anterior cruciate ligament reconstruction. Arthroscopy 2011;27(08):1129-1134

31 Ross MD, Irrgang JJ, Denegar CR, McCloy CM, Unangst ET. The relationship between participation restrictions and selected clinical measures following anterior cruciate ligament reconstruction. Knee Surg Sports Traumatol Arthrosc 2002;10 (01):10-19. Doi: 10.1007/s001670100238

32 Barenius B, Nordlander M, Ponzer S, Tidermark J, Eriksson K. Quality of life and clinical outcome after anterior cruciate ligament reconstruction using patellar tendon graft or quadrupled semitendinosus graft: an 8-year follow-up of a randomized controlled trial. Am J Sports Med 2010;38(08):1533-1541

33 Frobell RB, Roos EM, Roos HP, Ranstam J, Lohmander LS. A randomized trial of treatment for acute anterior cruciate ligament tears. N Engl J Med 2010;363(04):331-342

34 Meunier A, Odensten M, Good L. Long-term results after primary repair or non-surgical treatment of anterior cruciate ligament rupture: a randomized study with a 15 -year follow-up. Scand J Med Sci Sports 2007;17(03):230-237

35 Fithian DC, Paxton EW, Stone ML, et al. Prospective trial of a treatment algorithm for the management of the anterior cruciate ligament-injured knee. Am J Sports Med 2005;33(03): 335-346

36 Larkin JJ, Barber-Westin SD. The effect of injury chronicity and progressive rehabilitation on single-incision arthroscopic anterior cruciate ligament reconstruction. Arthroscopy 1998; 14(01):15-22

37 Anstey DE, Heyworth BE, Price MD, Gill TJ. Effect of timing of ACL reconstruction in surgery and development of meniscal and chondral lesions. Phys Sportsmed 2012;40(01):36-40. Doi: 10.3810/psm.2012.02.1949

38 Fok AW, Yau WP. Delay in ACL reconstruction is associated with more severe and painful meniscal and chondral injuries. Knee Surg Sports Traumatol Arthrosc 2013;21(04):928-933. Doi: 10.1007/s00167-012-2027-1

39 Smith TO, Davies L, Hing CB. Early versus delayed surgery for anterior cruciate ligament reconstruction: a systematic review and meta-analysis. Knee Surg Sports Traumatol Arthrosc 2010;18 (03):304-311

40 Andernord D, Karlsson J, Musahl V, Bhandari M, Fu FH, Samuelsson $\mathrm{K}$. Timing of surgery of the anterior cruciate ligament. Arthroscopy 2013;29(11):1863-1871. Doi: 10.1016/j.arthro.2013.07.270

41 Ardern CL, Österberg A, Tagesson S, Gauffin H, Webster KE, Kvist J. The impact of psychological readiness to return to sport and recreational activities after anterior cruciate ligament reconstruction. Br J Sports Med 2014;48(22):1613-1619. Doi: 10.1136/bjsports-2014-093842

42 Hsu CJ, Meierbachtol A, George SZ, Chmielewski TL. Fear of Reinjury in Athletes. Sports Health 2017;9(02):162-167. Doi: $10.1177 / 1941738116666813$

43 Dingenen B, Gokeler A. Optimization of the Return-to-Sport Paradigm After Anterior Cruciate Ligament Reconstruction: A Critical Step Back to Move Forward. Sports Med 2017;47(08): 1487-1500. Doi: 10.1007/s40279-017-0674-6

44 Kori S, Miller R, Todd D. Kinesiophobia: A new view of chronic pain behavior. Pain Manage 1990; **:35-43

45 Flanigan DC, Everhart JS, Pedroza A, Smith T, Kaeding CC. Fear of reinjury (kinesiophobia) and persistent knee symptoms are common factors for lack of return to sport after anterior cruciate ligament reconstruction. Arthroscopy 2013;29(08): 1322-1329. Doi: 10.1016/j.arthro.2013.05.015

46 Medvecky MJ, Nelson S. Kinesiophobia and Return to Sports After Anterior Cruciate Ligament Reconstruction. Conn Med 2015;79 (03):155-157

47 Nwachukwu BU, Adjei J, Rauck RC, et al. How Much Do Psychological Factors Affect Lack of Return to Play After Anterior Cruciate Ligament Reconstruction? A Systematic Review. Orthop J Sports Med 2019;7(05):2325967119845313. Doi: $10.1177 / 2325967119845313$

48 Miller RP, Kori SH, Todd DD. The Tampa Scale. Clin J Pain 1991; 7:51. Doi: 10.1097/00002508-199103000-00053

49 Burland JP, Toonstra J, Werner JL, Mattacola CG, Howell DM, Howard JS. Decision to Return to Sport After Anterior Cruciate Ligament Reconstruction, Part I: A Qualitative Investigation of Psychosocial Factors. J Athl Train 2018;53(05):452-463. Doi: 10.4085/1062-6050-313-16

50 Paterson FW, Trickey EL. Anterior cruciate ligament reconstruction using part of the patellar tendon as a free graft. 
10 Retorno Deportivo en Reconstrucción LCA: Factores Asociados Pakuts y col

J Bone Joint Surg Br 1986;68(03):453-457. Doi: 10.1302/0301620x.68b3.3733814

51 Sonesson S, Kvist J, Ardern C, Österberg A, Silbernagel KG. Psychological factors are important to return to pre-injury sport activity after anterior cruciate ligament reconstruction: expect and motivate to satisfy. Knee Surg Sports Traumatol Arthrosc 2017;25 (05):1375-1384. Doi: 10.1007/s00167-016-4294-8
52 Nagelli CV, Hewett TE. Should Return to Sport be Delayed Until 2 Years After Anterior Cruciate Ligament Reconstruction? Biological and Functional Considerations. Sports Med 2017;47 (02):221-232. Doi: 10.1007/s40279-016-0584-z

53 Whitehead TS. Failure of anterior cruciate ligament reconstruction. Clin Sports Med 2013;32(01):177-204. Doi: $10.1016 /$ j.csm.2012.08.015 